\title{
Modeling the Effects of Transforming Growth Factor-beta on Extracellular Matrix Alignment in Dermal Wound Repair
}

\author{
J. C. Dallon \\ dallon@math.byu.edu
}

\author{
J. A. Sherratt \\ P. K. Maini
}

Follow this and additional works at: https://scholarsarchive.byu.edu/facpub

Part of the Mathematics Commons

\section{Original Publication Citation}

J.C. Dallon, J.A. Sherratt, P.K. Maini: Modeling the Effects of Transforming Growth Factor-beta on Extracellular Matrix Alignment in Dermal Wound Repair. Wound Repair and Regeneration. 9(4): 278-286 (21).

\section{BYU ScholarsArchive Citation}

Dallon, J. C.; Sherratt, J. A.; and Maini, P. K., "Modeling the Effects of Transforming Growth Factor-beta on Extracellular Matrix Alignment in Dermal Wound Repair" (2001). Faculty Publications. 1082.

https://scholarsarchive.byu.edu/facpub/1082 


\section{Modeling the Effects of Transforming Growth Factor- $\beta$ on Extracellular Matrix Alignment in Dermal Wound Repair}

John C. Dallon ${ }^{1}$, PhD, Jonathan A. Sherratt ${ }^{2}, \mathrm{PhD}$ and Philip K. Maini ${ }^{3} \mathrm{PhD}$

${ }^{1}$ Department of Mathematics

Brigham Young University

Provo, UT 84602-6539 U.S.A.

${ }^{2}$ Department of Mathematics Heriot-Watt University Edinburgh, EH14 4AS U.K.

${ }^{3}$ Centre for Mathematical Biology

Mathematical Institute

University of Oxford 24-29 St Giles' Oxford OX1 3LB, U.K.

October 17, 2001 


\begin{abstract}
We present a novel mathematical model for collagen deposition and alignment during dermal wound healing, focusing on the regulatory effects of TGF $\beta$. Our work extends a previously developed model which considers the interactions between fibroblasts and extracellular matrix, composed of collagen and a fibrin based blood clot, by allowing fibroblasts to orient the collagen matrix, and produce and degrade the extracellular matrix, while the matrix can direct the fibroblasts and control their speed. Here we extend the model by allowing a time varying concentration of TGF $\beta$ to alter the properties of the fibroblasts. Thus we are able to simulate experiments which alter the TGF $\beta$ profile. Within this model framework we find that most of the known effects of $\operatorname{TGF} \beta$, i.e., changes in cell motility, cell proliferation and collagen production, are of minor importance to matrix alignment and cannot explain the anti-scarring properties of TGF $\beta$. However, we find that by changing fibroblast reorientation rates, consistent with experimental evidence, the alignment of the regenerated tissue can be significantly altered. This provides an explanation for the experimentally observed influence of TGF $\beta$ on scarring.
\end{abstract}

keywords: cell flux, cell speed, cell polarization, tissue regeneration, collagen production, fibrin degradation. 


\section{Introduction}

Typically wound repair results in a scar where the new regenerated tissue is distinctly different from the old (normal) tissue, and less functional. Recent studies show that altering the profile of transforming growth factor- $\beta$ or $\operatorname{TGF} \beta$ during healing can have profound effects on the healing process, including significantly increasing or decreasing the degree of scarring $[1,2,3,4]$. It is known that TGF $\beta$ alters the wound healing process in many ways $[5,6]$, and it is unclear which of these effects are responsible for changing the degree of scarring. The basic objective of this paper is to use mathematical modeling to determine those aspects of $\operatorname{TGF} \beta$ activity that are most significant for wound repair.

One of the main differences between scar tissue and normal tissue is the alignment of the extracellular matrix. For example, in normal rodent tissue the collagen fibers of the extracellular matrix are randomly aligned, whereas in scar tissue they are primarily aligned perpendicular to the basement membrane [7]. Other differences include higher density of collagen, thicker fibers, and lower tensile strength in scar tissue, as well as different ratios of the types of collagen present [8]. In this present paper, we are primarily concerned with the difference in alignment, which is currently thought to play a major role in determining scar quality [9].

The model simulates the invasion of the wound region by surrounding fibroblasts, which are guided by the extracellular matrix. They in turn modify the extracellular matrix, which for simplicity we assume to be composed only of fibrin and collagen, by degrading, producing and aligning the proteins. By using this mathematical model, which focuses on simple interactions between the fibroblasts and the extracellular matrix, we can easily manipulate the system in a manner unavailable in experiments, and can thus help to understand the complex dynamics of wound healing. Within this modeling framework, we can explore the consequences of TGF $\beta$-regulation of fibroblast properties.

The paper is organized in the following manner. The model is described in the next section and numerical simulations of wound repair are presented in $\S 3$. In $\S 4$ we conclude by discussing the implications of these simulations.

\section{The Model}

Our model is an extension of one presented previously [10], which considers three components: fibroblasts, collagen and fibrin. The new ingredient here is that we include explicitly the dependence of fibroblast properties on TGF $\beta$. The collagen and fibrin networks are described by continuous functions 
representing direction (the predominant local fiber direction) and density, and the fibroblasts move and modify these networks: we track separately the movement of each fibroblast cell. The concentration of $\operatorname{TGF} \beta$ is given by a function which varies with time but is constant throughout the wound region, which is taken to be a two-dimensional cross section of a slash wound. Typically it is 1 millimeter deep and 0.5 millimeters wide.

Within this framework we incorporate a range of cell-ECM interactions. The fibroblasts are guided by the extracellular matrix, which is composed of collagen and fibrin. This well-documented phenomenon is known as contact guidance $[11,12,13]$. In our model, we assume that as the cells move, they sample their environment, the extracellular matrix, and determine which direction to move in, based on the local fiber orientation see the appendix for details. Additionally, their speed is affected by the density of the different proteins. In particular, the fibroblast speed depends on fibronectin in a biphasic manner [14], with cells moving more slowly at low or high density. We are assuming that the fibronectin concentration is proportional to the fibrin concentration and that the densities are limited to the range where the speed increases. Thus we take the cell speed to increase linearly with fibrin concentration. The response to collagen density is similarly biphasic, but the cell speed is more sensitive to fibronectin [15] than to collagen. Specifically, we assume that the extracellular matrix can alter the cell's speed by a factor of about 8, with the average speed being between 2 to 5 microns per hour. The reader is referred to the appendix and previous work [10] for the precise functional forms and more details.

The cells are modeled to allow polarization, that is, their direction of motion is influenced by their previous direction. The cells enter the wound region from the surrounding tissue and proliferate by cell division. Experiments suggest that most of the fibroblasts in the wound are derived from the subcutaneous fascia [16] and in most simulations we assume that 80 percent of the cells entering the wound from surrounding tissue come from the fascia. The total number of cells in the wound region varies with time. Thus the proportion which migrite from the surrounding tissue and the proportion of cells due to cell proliferation depend on time and the proliferation rate.

Fibroblast behavior is determined by the extracellular matrix, but the cells also modify the extracellular matrix around them. Thus in our model the cells degrade collagen and fibrin as well as produce collagen. The simulated cells degrade collagen (and fibrin) at a rate proportional to the amount of collagen (or fibrin) already present, and produce collagen at a constant rate, oriented in the direction of movement. This allows us to simulate the transformation, via fibroblasts, of a fibrin blood clot into 
extracellular matrix composed primarily of collagen. As the cells change the composition of the extracellular matrix, they also align the matrix $[17,18]$. It is unclear whether this alignment is due only to mechanical forces the cells exert on the matrix as they move [19], or other more local influences due to the production of new fibers. In our model we do not distinguish between these different effects: we simply assume alignment is a local effect, in which the fibroblasts align the collagen matrix (but not fibrin) towards the direction in which they are moving.

There are many sources of TGF $\beta$ in the wound including monocytes, fibroblasts and platelets, which seem to be the earliest source [5]. In view of this range of sources, and because the wound region is relatively small with no experimental data to quantify spatial variations, we assume a uniform concentration of TGF $\beta$ in the wound region which varies with time.

It is known that TGF $\beta$ has various effects on fibroblasts: it acts as a chemoattractant, modulates collagen production and degradation as well as cell proliferation [20,21]. In our mathematical model it is easy to incorporate all of these effects, but more importantly we can separate them, and study how they relate to one another. In the remainder of this paper we give results of simulations which examine the individual effects of $\operatorname{TGF} \beta$, the effects of different isoforms of $\operatorname{TGF} \beta$, and finally we end with a discussion of our findings. But we begin by giving further details about the model.

\section{Results}

Our study of TGF $\beta$ activity is made possible by the recent data of Yang and coworkers [22], who have determined a detailed time-course of active TGF $\beta$ in dermal wound healing in rats. They found that the concentration of active TGF $\beta$ peaks within an hour after wounding, decreases to a level above normal within one day, peaks again five days after wounding and then more gradually decreases until reaching approximately normal levels 14 days after wounding. Since fibroblasts do not enter the wound until 24 to 48 hours after wounding, we ignore the first peak in TGF $\beta$ concentration and use the functional form shown in Figure 1 for the TGF $\beta$ concentration.

We begin by considering separately the effects of TGF $\beta$ on cell proliferation, cell motility and collagen production. 


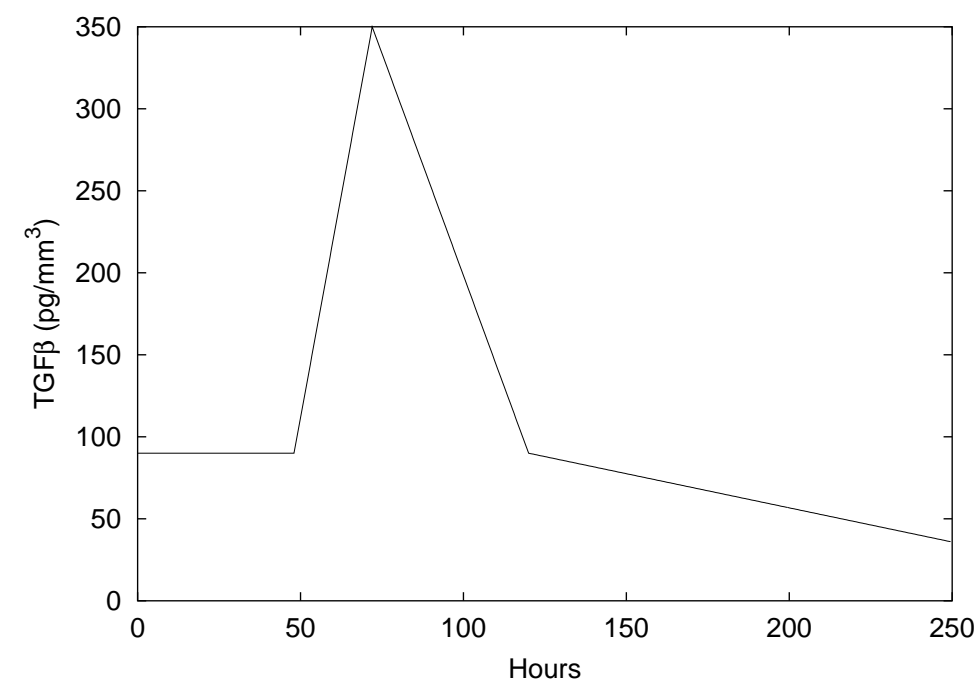

Figure 1. A graph of the function used for the TGF $\beta$ concentration. Time $t=0$ corresponds to 48 hours after wounding, which is when fibroblasts begin entering the wound. This graph is based on data taken from Yang et. al. [22].

\subsection{The effect of TGF $\beta$ on cell proliferation}

The effect of TGF $\beta$ on cell proliferation depends on the developmental age of the source of the fibroblasts, with fibroblasts from younger sources having a greater proliferative response to the growth factor ranging from just over 100 percent of normal to over 200 percent of normal [23, 24]. The response depends on concentration in a biphasic manner, increasing and then decreasing with concentration, with the maximum response of over 250 percent above normal at a concentration of $1 \mathrm{pM}$ in monolayers [20]. We allow the fibroblast proliferation to vary with $\operatorname{TGF} \beta$ concentration in a linear manner, increasing the proliferation up to a maximum of two times that of normal. This results in a change from 66 percent of the total number of cells at the end of the simulation resulting from cell proliferation to 77 percent. Previous investigations [10] showed that greatly increased cell division tended to randomize the fiber alignment due to a decrease in the number of cells entering from the surrounding tissue (the maximum cell density is kept constant), and due to our assumption that cells lose their polarization when they divide. However, our simulations show that the relatively slight increases (a factor of 2 at most) in cell proliferation caused by $\operatorname{TGF} \beta$ has little effect on the fiber alignment. The density distribution of the wound region changes, with more dense collagen in the center towards the base of the wound: since the cells more rapidly accumulate in the wound region when there is increased proliferation, the overall collagen density is typically higher at any given time post-wounding.

The response to $\operatorname{TGF} \beta$ is biphasic and the proliferation is less at the highest concentrations reported, 


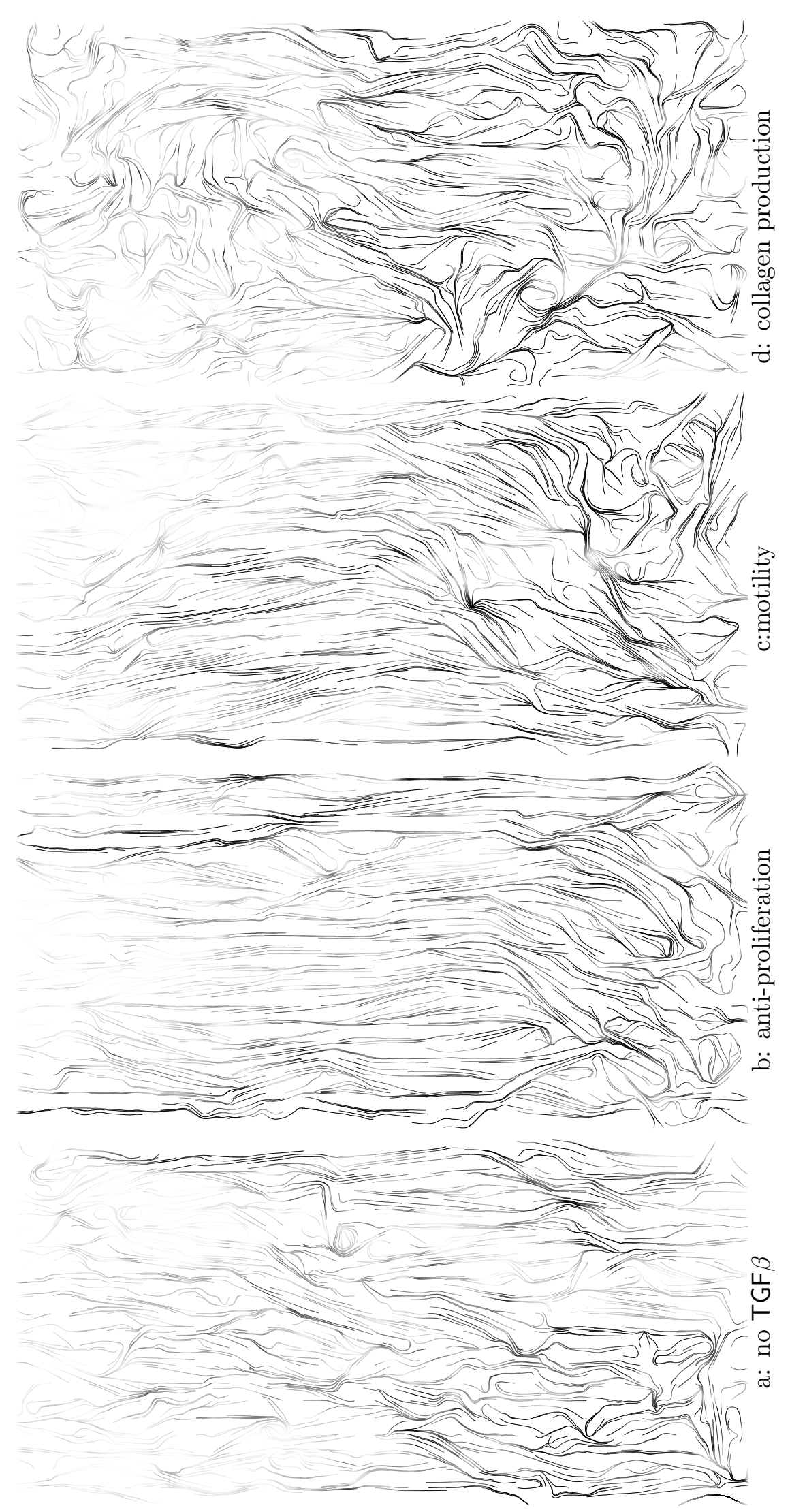

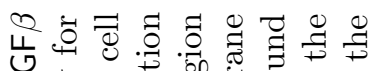

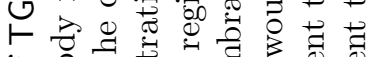
फै

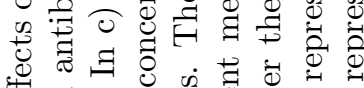

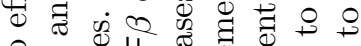

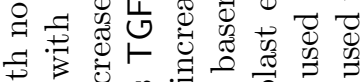

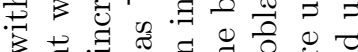
$\infty 0$ d

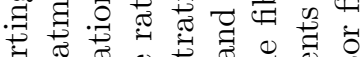

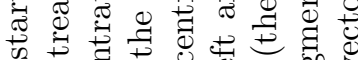
F 웡 s 0 . ज

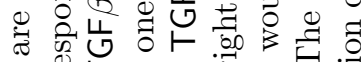
Q

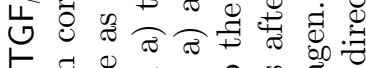

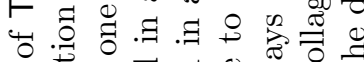

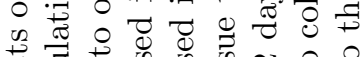

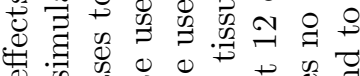
क्व.

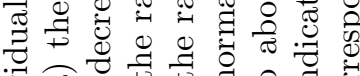

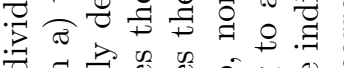

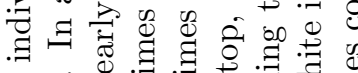
宁品

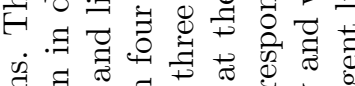
पू

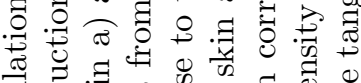

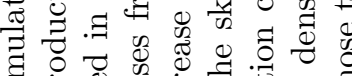

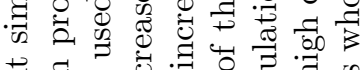
궁 过

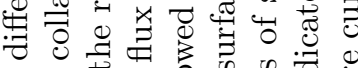

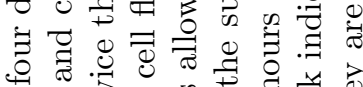
政官

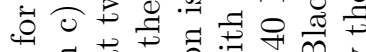
몌

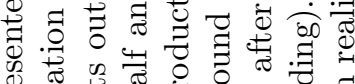

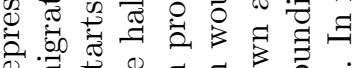

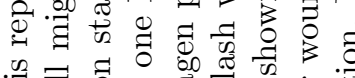
न

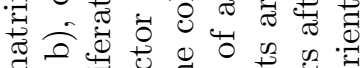

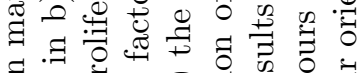
व $\stackrel{0}{=}$. O

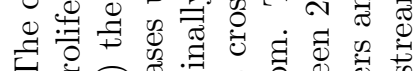

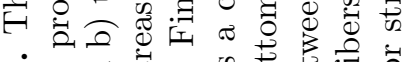
ง

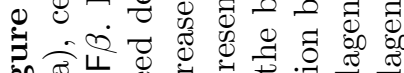

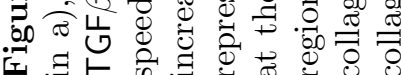




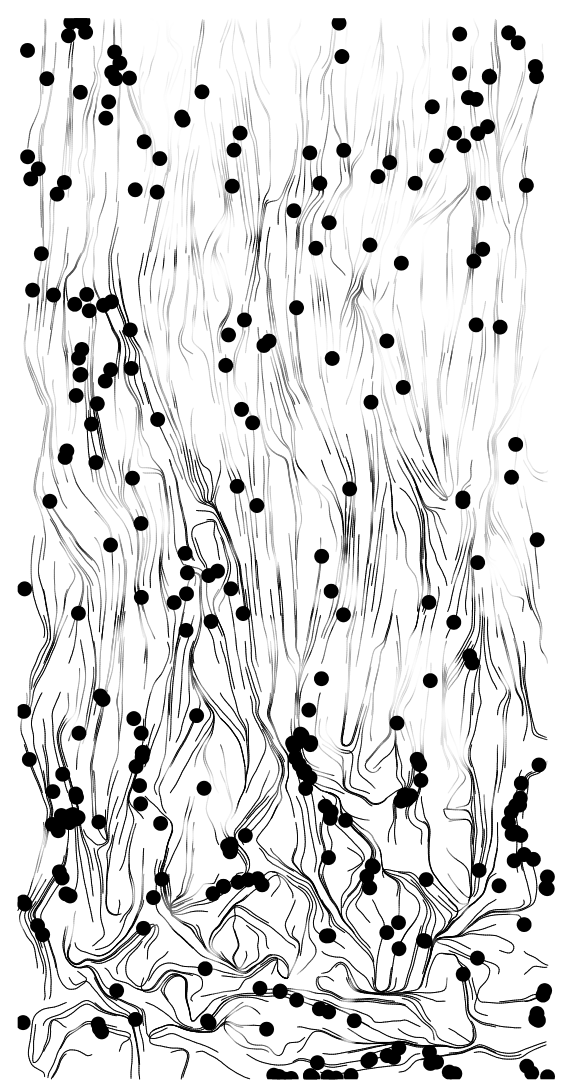

a: normal

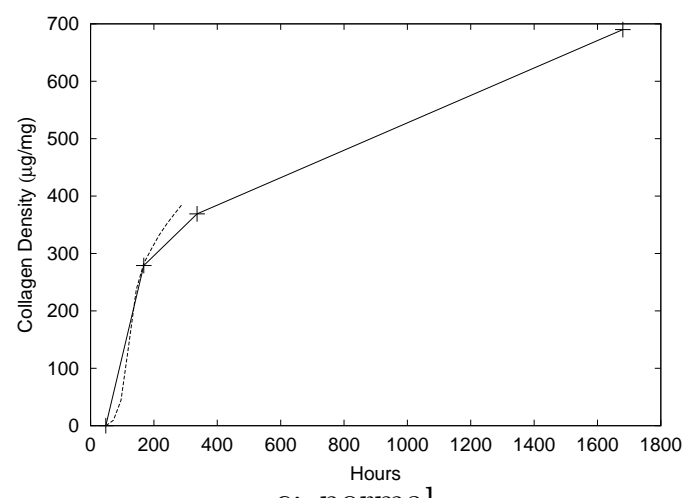

c: normal
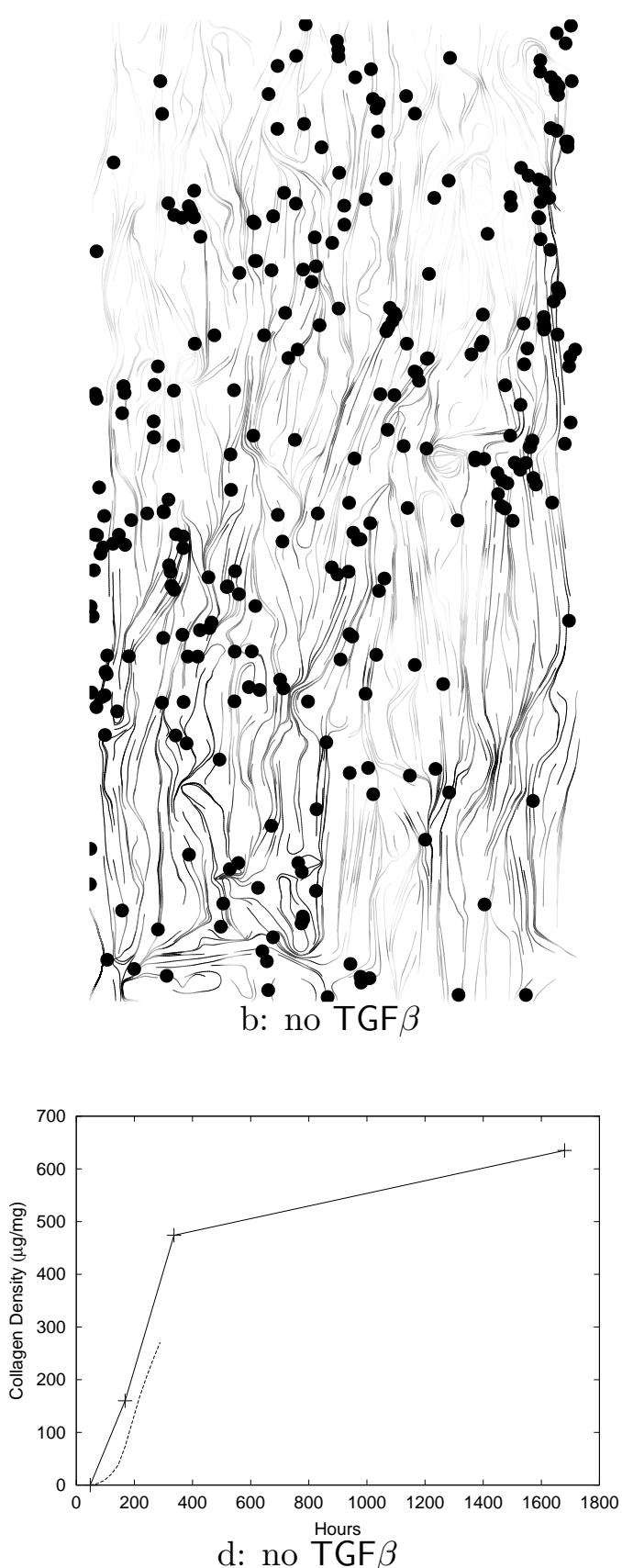

Figure 3. The collagen matrix for a simulation of normal wound healing with TGF $\beta$, resulting in a scar, is shown in a) with the fibroblast locations marked and graphs of total collagen density for the simulation and the experimental control data [1] are plotted in c). In b) we show a simulation of a wound treated with an antibody for TGF $\beta$ with the corresponding collagen density for the simulation and the experimental data plotted in d). The anti TGF $\beta$ simulation is the same as that shown in figure 2a) and removes only the effects of TGF $\beta$ on cell proliferation, motility and collagen production shown individually in figure 2. The simulation clearly shows that those three effects do not explain the experimental data where anti TGF $\beta$ treatment reduces scarring. The collagen matrix is represented after 240 hours of simulation corresponding to about 12 days after wounding (the fibroblasts enter the wound region between 24-48 hours after wounding). Black indicates high density and white indicates no collagen. The line segments are streamlines for the collagen vector field. The location of one fourth of the total fibroblasts are plotted with black circles. The region represents a cross section of a slash wound with the surface of the skin at the top, normal tissue to the right and left and the basement membrane at the bottom. When combining the three effects of TGF $\beta$, we assume that the cell proliferation increases at $90 \mathrm{pg} / \mathrm{mm}^{3}$ of TGF $\beta$ and then decreases for higher concentrations; for the motility we combine the decreased cell speed and the elevated flux at $90 \mathrm{pg} / \mathrm{mm}^{3}$ of TGF $\beta$ which decreases with increased concentrations of TGF $\beta$. The three experimental values in c) and d) are denoted by $(+)$ and are averaged values of four observations reported in [1]. The simulated collagen density is plotted by the dashed line. 
which are of the same order of magnitude as the concentration reported in the wound, so we must consider the possibility that proliferation decreases as the TGF $\beta$ concentration peaks. Thus, in contrast to our previous simulation, we begin with a proliferation rate 2 times higher than normal, decreasing with the increasing TGF $\beta$ concentration. This is consistent with the experimentally measured cell proliferation in the wound region, which falls dramatically between day 5 and day 7 post-wounding [16]. Surprisingly, this results in more alignment of the collagen fibers (see figure $2 b$ ). In this simulation the cells do not get slowed down in high density regions near the boundary of the wound, and thus are more uniformly distributed thoroughout the wound region, leading to increased alignment.

\subsection{The effect of TGF $\beta$ on cell motility}

As in the case of cell proliferation, the experimental evidence does not indicate a simple relationship between fibroblast motility and TGF $\beta$ levels. Fibroblasts show a biphasic response to TGF $\beta$ concentrations, and the maximum response involves 400 percent more cells migrating than normal [20]. Further, it was reported that this increased migration was not due to chemokinesis but was a chemotactic response: that is, there is not an increase in overall motility, but a directed movement along chemical gradients. In other work where fibroblasts are placed on 3-dimensional collagen gels and their motility is studied by examining how many invade the gel, the effect of TGF $\beta$ again depends on the developmental age of the source of fibroblasts as well as the cell density [25, 26]. It was found that at low densities half as many adult fibroblasts migrated into the gel with increased TGF $\beta$ concentration. In this study TGF $\beta$ was uniformly increased so there were no chemical gradients. The results from both of these studies suggest that TGF $\beta$ causes a chemotatic response and alters the speed of the cells. In our model we alter those two components: we linearly decrease the cell speed up to a factor of one half depending on the TGF $\beta$ concentration, and we increase the cell flux into the wound region by up to a factor of four, again depending on the TGF $\beta$ concentration. Cell speed can have significant effects on collagen alignment, because in the model, cells move in one direction for a fixed time interval, before (potentially) reorienting. Thus when the cells move faster, they move a greater distance in a particular direction during this fixed time interval, causing more alignment [10]. In this case the speed decrease is relatively slight, and causes the collagen to be only slightly less aligned. In contrast, the increased flux significantly increases alignment perpendicular to the skin surface, since 80 percent of the cells are assumed to enter the wound from underlying tissue, and only $20 \%$ from the surrounding dermis [16]. Combining both effects gives a net increase in alignment, since the alignment due to the flux overcomes the randomizing influence of the decreased speed. Since the chemotactic response is biphasic, we also 
ran a simulation where the peak in $\operatorname{TGF} \beta$ concentration decreased the cell flux from the elevated levels. That simulation gave results that were similar to the increasing flux case, but with slightly more alignment (see figure 2c).

\subsection{The effect of TGF $\beta$ on collagen production}

Because the speed of the fibroblasts depends on the collagen density, changes in collagen production rate affect the speed of the cells and therefore the alignment of the collagen fibers. By increasing collagen production [27] and decreasing collagenase production [28], TGF $\beta$ modifies the overall extracellular collagen density [29]. At TGF $\beta$ concentrations comparable to those found in wounds, the overall collagen accumulation is increased by a factor of two [30]. At lower concentrations, the increase in collagen was found to range from two-to-three fold and was dependent on the amount of collagen already present in the medium [21]. In our model simulations we allow the collagen production to increase linearly to a maximum of three times normal production. This results in a less aligned collagen gel since, as the overall collagen density is higher, the cells are slightly slower and the reduced speed decreases the alignment (see figure $2 \mathrm{~d}$ ).

\subsection{Summary of the results presented above}

Taken together these various results show that the effects of $\operatorname{TGF} \beta$ on cell proliferation, movement and collagen production are unable to explain the experimentally observed collagen alignment patterns. This is illustrated in Figure 3a,c, which shows the combined effect of these three regulatory functions of $\operatorname{TGF} \beta$. Moreover, corresponding computer simulations in which the TGF $\beta$ concentration is set to zero (see figure $3 \mathrm{~b}, \mathrm{~d}$ ) show a net increase in alignment, in contrast to the observed reduction in scarring in wounds treated with antibodies for $\operatorname{TGF} \beta$. This indicates that other regulatory functions of TGF $\beta$ must be the basic cause of its effect on wound repair.

\subsection{The effect of TGF $\beta$ on cell reorientation}

Recent experimental evidence indicates that $\mathrm{TGF} \beta$ controls cell movement in a more complicated manner than simply controlling the speed of movement. The development of lamellipodia and filopodia by fibroblasts is dependent on TGF $\beta$ levels, implying control of the frequency with which cells change direction [31]. There is currently no quantitative experimental data on the extent of this effect, but 
nevertheless we can study its qualitative implications using the model. Figure 4 shows a simulation where the cells are allowed to reorient three times more frequently than those shown in figure 3b: approximately every 9 minutes. This significantly alters the resulting alignment, in a manner that agrees with experimentally observed alignment patterns.

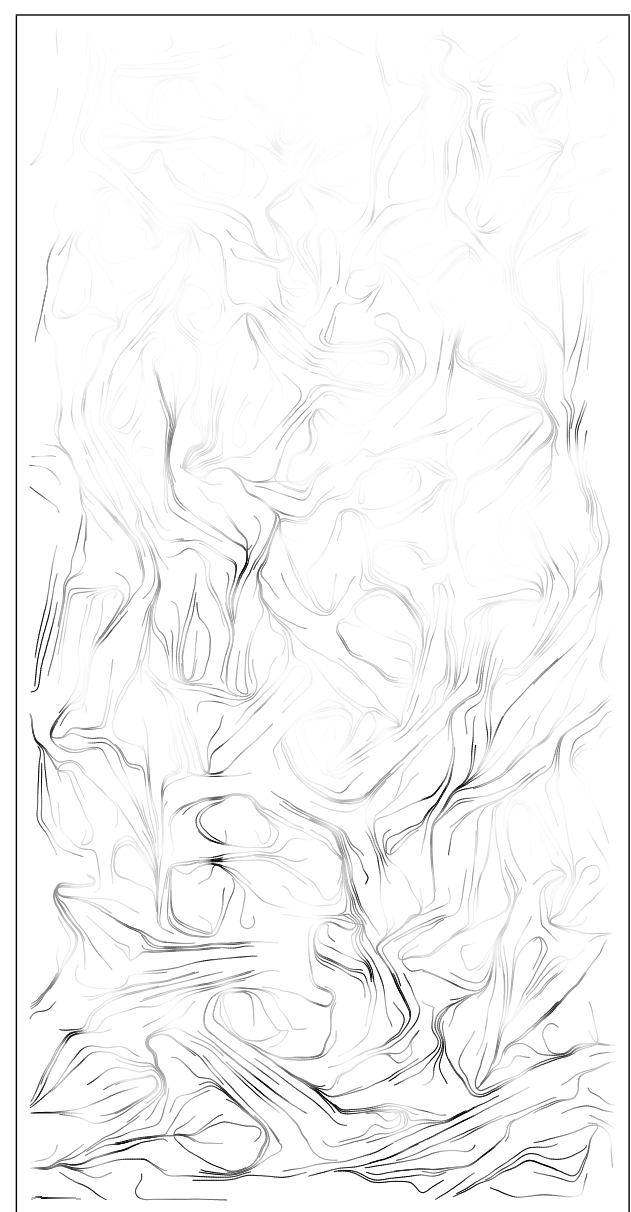

a

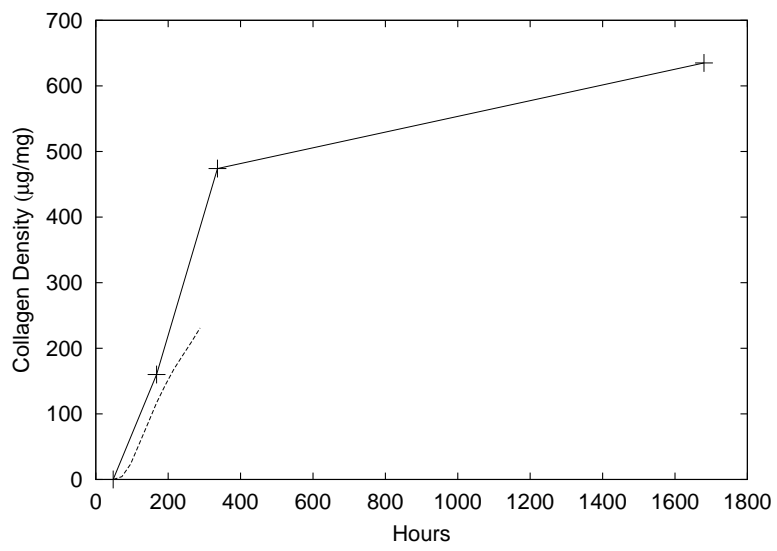

$\mathrm{b}$

Figure 4. Part a) represents the collagen matrix for a numerical experiment simulating anti TGF $\beta_{1}$ treatment, showing a more disordered collagen fiber pattern that is highly reminiscent of experimental data. The more disordered matrix pattern is a key component of reduced scarring. The parameters for this simulation are identical to figure $3 \mathrm{~b}$ except the cells reorient approximately every 9 minutes instead of every 27 minutes. Graphs of total collagen density for the simulation and from experimental data [1] are plotted in b). The collagen matrix is represented after 240 hours of simulation corresponding to about 12 days after wounding (the fibroblasts enter the wound region between 24-48 hours after wounding). Black indicates high density and white indicates no collagen. The region represents a cross section of a slash wound with the surface of the skin at the top, normal tissue to the right and left and the basement membrane at the bottom. The line segments are streamlines for the collagen vector field. The three experimental values in b) are denoted by $(+)$ and are averaged values of four observations reported in [1]. The simulated collagen density is plotted by the dashed line. 


\section{Different Isoforms of TGF $\beta$}

Three isoforms of TGF $\beta$ are found in mammals; TGF $\beta_{1}, \operatorname{TGF} \beta_{2}$ and $\operatorname{TGF} \beta_{3}$ [29]. By neutrilizing TGF $\beta_{1}$ and TGF $\beta_{2}$ in a wound or by adding $\operatorname{TGF} \beta_{3}$, scarring is reduced [3]. Interestingly, wounds in transgenic mice overexpressing TGF $\beta_{1}$ healed with less scarring [4]; this finding is consistent with previous results since explicit measurements indicate that in comparison to normal tissue, wound levels are higher for $\operatorname{TGF} \beta_{3}$, and lower for $\operatorname{TGF} \beta_{1}$. Most experimental evidence suggests that the three isoforms affect fibroblasts similarly [30, 20]. There are two notable exceptions: Murata and coworkers [32] found that $\mathrm{TGF} \beta_{3}$ increases TGF $\beta_{1}$ production and alters collagen production in a manner dependent on TGF $\beta_{1}$. Incorporating this into our model would not be difficult, but altering the collagen production rate by the magnitude consistent with the experiments would not result in significant differences in alignment. The second exception is recent experimental evidence which suggests that TGF $\beta_{3}$ increases the number of filopodia of mesenchymal cells while $\mathrm{TGF} \beta_{1}$ and $\mathrm{TGF} \beta_{2}$ promote the formation of lamellipodia [31]. In this study cells from TGF $\beta_{3}$ knockout mice show a third less filopodia than normal cells from the palatal shelf and when TGF $\beta_{3}$ is added, the knockout cells show 3 to 5 times more filopodia. An increase in the number of filopodia of a cell will cause it to have a better sampling of its environment and will thus tend to increase its probability of moving in other directions. This is because if the filopodia are extended with increased frequency or there are more filopodia for a greater period of time the cell may reorient more frequently. This provides a clear explanation for the anti-scarring effects of TGF $\beta_{3}$ : the experimental data suggests that adding TGF $\beta_{3}$ will tend to cause more frequent cell reorientation, and our mathematical model shows that this will lead to a more disordered network of collagen fibers, which is a hallmark of reduced scarring. Antibodies to TGF $\beta_{1}$ and TGF $\beta_{2}$, on the other hand, will tend to reduce lamellipodia formation. However, there will also be increased binding of $\mathrm{TGF} \beta_{3}$ to the cell surface, since it is likely that to some degree the isoforms bind competitively [33, 34], and we anticipate that the increase in filopodia formation due to this increased TGF $\beta_{3}$ will be the dominant effect, leading to reduced scarring - again as observed experimentally.

\section{Discussion}

Over the last decade, a number of mathematical models have been developed, to represent different aspects of wound healing. These include models focusing on wound contraction [35, 36, 37], collagen composition [38], epidermal healing [39, 40, 41], alignment [42, 43] and angiogenesis [44, 45]. Moreover 
there is extensive modelling of closely related biological processes, including tissue mechanics [46], alignment $[47,48]$ and tumor growth and angiogenesis [49, 50, 51]. Although some of this previous work does focus on alignment, the representation of the alignment of dermal tissue in our previous work [10], on which this model is based, uses novel techniques, in particular the representation of fibroblast cells as discrete objects. In addition, in this paper we model the effects of TGF $\beta$ on alignment, which to our knowledge has not be done before.

We have presented a mathematical model for scar formation during wound repair that enables a detailed investigation of the effects on collagen fiber orientation of underlying biological processes. By incorporating the role of TGF $\beta$ into the model, we conclude that its effects on cell proliferation, migration (meaning speed and flux) and extracellular matrix production influence the collagen fiber alignment, which is a key indicator of scar quality [9], in only minor ways. Hence some other effect is needed to alter alignment if we are to explain its scar reducing properties. We predict that the recently-identified regulation by $\mathrm{TGF} \beta_{3}$ of filopodia extensions could be the crucial property of the TGF $\beta$ family, which underlies its ability to regulate scar tissue formation.

Due to the biphasic properties of $\operatorname{TGF} \beta$, careful time evaluation measurements of this cytokine need to be made and the possibility of significant spatial variation in concentration across the wound must be investigated. For example, we believe that the peak in TGF $\beta$ concentration five days after wounding is high enough to suppress fibroblast proliferation, while the elevated levels earlier in wounding increase proliferation. This is consistent with experimental evidence [16] where it was found that cell proliferation decreased dramatically between 5 and 7 days after wounding. The concentrations of TGF $\beta$ used in most experiments are well below the levels measured in the wound by Yang et. al. [22].

Figure 4 shows a simulation of the anti-TGF $\beta_{1}$ treatment and one can see that the model predicts much less alignment than for the normal wound healing case, which is shown in Figure 3a. Simulations of adding TGF $\beta_{3}$ are very similar: again there is increased cell reorientation, but the other effects of TGF $\beta$ (cell proliferation and speed, and collagen production) are retained. The effects of TGF $\beta$ on cell processes other than reorientation are less significant to alignment, and reorientation is affected differently by the different isoforms, which bind to the cells competitively. Thus our model predicts that it is the ratio of the concentrations of $\operatorname{TGF} \beta_{1,2}$ to $\operatorname{TGF} \beta_{3}$ that is the key issue in the regulation of fiber alignment within scar tissue. Quantitative data on the dynamics of these isoforms within a healing wound would be key additional information in the further development of the model.

Our model predicts that when the cells reorient every 30 minutes there is pronounced alignment, 
orientation every 15 minutes gives significant improvement (less alignment) and orientation every 10 minutes gives highly disordered tissue. When more experimental evidence is gathered, quantifying the effects of the TGF $\beta$ isoforms on orientation, our model could be used to obtain predictions in the form of dose-response curves for the relationship between the concentration of TGF $\beta$ isoforms and the degree of fiber alignment within scar tissue. 
Acknowledgments We are very grateful to Prof Mark Ferguson for helpful discussions. This work was supported in part by EPSRC grant GR/K71394. JAS was supported by SHEFC Research Development Grant 107, and by an Advanced Research Fellowship from EPSRC.

\section{References}

[1] Shah M, Foreman DM, Ferguson MWJ, Control of scarring in adult wounds by neutralising antibody to transforming growth factor $\beta$. Lancet, 1992;339:213-214

[2] Shah M, Foreman DM, Ferguson MWJ, Neutralising antibody to TGF- $\beta_{1,2}$ reduces cutaneous scarring in adult rodents. J Cell Sci, 1994;107:1137-1157

[3] Shah M, Foreman DM, Ferguson MWJ, Neutralisation of TGF- $\beta_{1}$ and TGF- $\beta_{2}$ or exogenous addition of TGF- $\beta_{3}$ to cutaneous rat wounds reduce scarring. J Cell Sci, 1995;108:985-1002

[4] Shah M, Revis DJ, Herrick S, Baillie R, Thorgeirson S, Ferguson M, Roberts A, Role of elevated plasma transforming growth factor- $\beta 1$ levels in wound healing. Am J Pathol, 1999;154(4):1115-1124

[5] Roberts AB, Sporn MB, Transforming growth factor- $\beta$. In: Clark RAF, editor, The Molecular and Cellular Biology of Wound Repair, Plenum Press, 2 edn., 1996; 275-308, 275-308

[6] O'Kane S, Ferguson MWJ, Transforming growth factor $\beta$ s and wound healing. Int J Biochem Cell Biol, 1997;29(1):63-78

[7] Whitby DJ, Ferguson MWJ, The extracellular matrix of lip wounds in fetal, neonatal and adult mice. Dev, 1991;112:651-668

[8] Ehrlich PH, Krummel TM, Regulation of wound healing from a connective tissue perspective. Wound Repair Regen, 1996;4:203-210

[9] Bowes LE, Jimenez MC, Hiester ED, Sacks MS, Brahmatewari J, Mertz P, Eagelstein WH, Collagen fiber orientation as quantified by small angle light scattering in wounds treated with transforming growth factor-beta(2) and its neutalizing antibody. Wound Repair Regen, 1999;7(3):179-186

[10] Dallon JC, Sherratt JA, Maini PK, Mathematical modelling of extracellular matrix dynamics using discrete cells: Fiber orientation and tissue regeneration. J Theor Biol, 1999;199(4):449-471

[11] Dunn GA, Ebendal T, Contact guidance on oriented collagen gels. Exp Cell Res, 1978;111:475-479 
[12] Guido S, Tranquillo RT, A methodology for the systematic and quantitative study of cell contact guidance in oriented collagen gels. J Cell Sci, 1993;105:317-331

[13] Oakley C, Jaeger NAF, Brunette DM, Sensitivity of fibroblasts and their cytoskeletons to substratum topographies: Topographic guidance and topographic compensation by micromachined grooves of different dimensions. Exp Cell Res, 1997;234:413-424

[14] Palecek SP, Loftus JC, Ginsberg MH, Lauffenburger DA, Horwitz AF, Integrin-ligand binding properties govern cell migration speed through cell-substratum adhesiveness. Nature, 1997;385:537540

[15] Wojciak-Stothard B, Denyer M, Mishra M, Brown RA, Adhesion orientation, and movement of cells cultured on ultrathin fibronectin fibers. In Vitro Cell Devel Biol, 1997;33(2):110-117

[16] Adams JJ, The cell kinetics of murine incisional wound healing. Ph.D. thesis, University of Manchester, 1997

[17] Trelstad RL, Hayashi K, Tendon collagen fibrillogenesis: Intracellular subassemblies and cell surface changes associated with fibril growth. Dev Biol, 1979;71:228-242

[18] Birk DE, Trelstad RL, Extracellular compartments in tendon morphogenesis: Collagen, fibril, bundle, and macroaggregate formation. J Cell Biol, 1986;103:231-240

[19] Harris AK, Stopak D, Wild P, Fibroblast traction as a mechanism for collagen morphogenesis. Nature, 1981;290:249-251

[20] Cordeiro MF, Bhattacharya SS, Schultz GS, Khaw PT, Tgf- $\beta 1,-\beta 2$, and - $\beta 3$ in vitro: Biphasic effects on tenon's fibroblast contraction, proliferation, and migration. Invest Ophth Vis Sci, 2000; 41(3):756-763

[21] Clark RAF, Nielsen LD, Welch MP, McPherson JM, Collagen matrices attenuate the collagensynthetic response of cultured fibroblasts to TGF- $\beta$. J Cell Sci, 1995;108:1251-1261

[22] Yang L, Qiu CX, Ludlow A, Ferguson MWJ, Brunner G, Active transforming growth factor- $\beta$ in wound repair. Am J Pathol, 1999;154(1):105-111

[23] Evangelisti R, V V, Bosi G, Baroni T, Bellucci C, Carinci P, Comparative effects of TGF $\beta$ on proliferation on 7- and 14-day-old chick embryo fibroblasts and lack of involvement of the odc/pa system in the TGF $\beta$ signaling pathway. J Cell Physiol, 1999;178:304-310 
[24] Kishi K, Nakajima H, Tajima S, Differential responses of collagen and glycosaminoglycan syntheses and cell proliferation to exogenous transforming growth factor $\beta 1$ in the developing mouse skin fibroblasts in culture. Brit J Plast Surg, 1999;52:579-582

[25] Ellis I, Schor SL, Differential effects of TGF- $\beta 1$ on hyaluronan synthesis by fetal and adult skin fibroblasts: Implications for cell migration and wound healing. Exp Cell Res, 1996;228:326-333

[26] Ellis I, Grey AM, Schor AM, Schor SL, Antagonistic effects of TGF- $\beta 1$ and MSF on fibroblast migration and hyaluronic acid synthesis. J Cell Sci, 1992;102:447-456

[27] Ignotz RA, Massague J, Tranforming growth factor- $\beta$ stimulates and the expression of fibronectin and collagen and their incorporation into the extracellular matrix. J Biol Chem, 1986;262(9):43374345

[28] Overall CM, Wrana JL, Sodek J, Independent regulation of collagenase, 72-kDa progelatinase, and metalloendoproteinase inhibitor expression in human fibroblasts by transforming growth factor- $\beta$. J Biol Chem, 1989;264(3):1860-1869

[29] Grande JP, Role of transforming growth factor- $\beta$ in tissue injury and repair. P Soc Exp Biol Med, 1997;214(1):27-40

[30] Locci P, Baroni T, Lilli C, Martinese D, Marinucci L, Bellocchio S, Calvitti M, Becchetti E, TGF $\beta$ and TGF $\alpha$, antagonistic effect in vitro on extracellular matrix accumulation by chick skin fibroblasts at two distinct embryonic stages. Int J Dev Biol, 1999;43:157-165

[31] Taya Y, O'Kane S, Ferguson MWJ, Pathogenesis of cleft palate in TGF- $\beta 3$ knockout mice. Dev, 1999;126:3869-3879

[32] Murata H, Zhou L, Ochoa S, Hasan A, Badiavas E, Falanga V, TGF- $\beta 3$ stimulates and regulates collagen synthesis through TGF- $\beta 1$-dependent and independent mechanisms. J Invest Dermatol, 1997;108:258-262

[33] Altomonte M, Montagner R, Fonsatti E, F C, Cattarossi I, Brasoveanu LI, Nicotra MR, Cattelan A, Natali PG, Maio M, Expression and structural features of endoglin (cd105), a transforming growth factor $\beta 1$ and $\beta 3$ binding protein, in human melanoma. Brit J Cancer, 1996;74:1586-1591

[34] Piek E, Heldin C, Kijke PT, Specificity, diversity, and regulation in TGF- $\beta$ superfamily signaling. FASEB, 1999;13:2105-2119 
[35] Tranquillo RT, Murray JD, Continuum model of fibroblast-driven wound contraction: Inflammation-mediation. J Theor Biol, 1992;158:135-172

[36] Olsen L, Sherratt JA, Maini PK, A mechanochemical model for adult dermal wound contraction and the permanence of the contracted tissue displacement profile. J Theor Biol, 1995;177:113-128

[37] Tracqui P, Woodward DE, Cruywagen GC, Cook J, Murray JD, A mechanical model for fibroblastdriven wound healing. J Biol Systems, 1995;3:1075-1084

[38] Dale PD, Sherratt JA, Maini PK, A mathematical model for collagen fibre formation during foetal and adult dermal wound healing. Proc R Soc Lond B, 1996;263:653-660

[39] Sherratt JA, Murray JD, Epidermal wound healing: The clinical implications of a simple mathematical model. Cell Transpl, 1992;1:365-371

[40] Dale P, Maini PK, Sherratt JA, Mathematical modeling of corneal epithelial wound healing. Math Biosci, 1994;124:127-147

[41] Gaffney EA, Maini PK, Sherratt JA, Tuft S, The mathematical modelling of cell kinetics in corneal epithelial wound healing. J Theor Biol, 1999;197(1):15-40

[42] Olsen L, Sherratt JA, Maini PK, Marchant B, Simple modelling of extracellular matrix alignment in dermal wound healing I. cell flux induced alignment. J Theor Med, 1998;1:172-192

[43] Olsen L, Maini PK, Sherratt JA, Dallon JC, Mathematical modelling of anisotropy in fibrous connective tissue. Math Biosci, 1999;158:145-170

[44] Olsen L, Sherratt JA, Maini PK, Arnold F, A mathematical model for the capillary endothelial cell-extracellular matrix interactions in wound-healing angiogenesis. IMA J Math Appl Med Biol, 1997;14:261-281

[45] Pettet GJ, Chaplain MAJ, McElwain DLS, , Byrne HM, On the role of angiogenesis in wound healing. Proc R Soc Lond B, 1996;263:1487-1493

[46] Barocas VH, Tranquillo RT, An anisotropic biphasic theory of tissue-equivalent mechanics: the interplay among cell traction, fibrillar network deformation, fibril alignment and cell contact guidance. AMSE J Biomech Eng, 1997;119:137-145

[47] Edelstein-Keshet L, Ermentrout BG, Models for contact-mediated pattern formation: cells that form parallel arrays. J Math Biol, 1990;29:33-58 
[48] Dallon JC, Sherratt JA, A mathematical model for fibroblast and collagen orientation. Bull Math Biol, 1998;60(1):101-129

[49] Anderson ARA, Chaplain MAJ, Continuous and discrete mathematical models of tumor-induced angiogenesis. Bull Math Biol, 1998;60(5):857-899

[50] Chaplain MAJ, Byrne HM, Mathematical modelling of wound healing and tumour growth - 2 sides of the same coin. Wounds: A Compendium of Clinical Research and Practice, 1996;8:587-616

[51] Holmes M, Sleeman B, A mathematical model of tumour angiogenesis incorporating cellular traction and viscoelastic effects. J Theor Biol, 2000;202(2):95-112 


\section{Appendix}

In this appendix the model equations used in the simulations are described. For more details the reader is referred to [10]. The collagen matrix and fibrin clot (blood clot) are denoted $\mathbf{c}(\mathbf{x})$ and $\mathbf{b}(\mathbf{x})$. These are all vectors in $\mathrm{R}^{2}$ which depend on time and space. The direction of the vector represents the predominant orientation of the fiber and the length of the vector gives the density of the protein. The fibroblast paths are denoted by $\mathbf{f}^{i}$ respectively $(i=1,2, \ldots, M)$. The equation determining $\mathbf{f}^{i}$ is given by

$$
\begin{aligned}
\mathbf{f}^{i}(t)^{\prime} & =s\left(\left\|\mathbf{c}\left(\mathbf{f}^{i}(t)\right)\right\|,\left\|\mathbf{b}\left(\mathbf{f}^{i}(t)\right)\right\|, g\right) \frac{\mathbf{u}(t)}{\|\mathbf{u}(t)\|} \\
\mathbf{u}(t) & =(1-\rho) \frac{\mathbf{v}^{i}(t)}{\left\|\mathbf{v}^{i}(t)\right\|}+\rho \frac{\mathbf{f}^{i}(t-\tau)^{\prime}}{\left\|\mathbf{f}^{i}(t-\tau)^{\prime}\right\|} \\
\mathbf{v}^{i}(t) & =(1-\alpha) \mathbf{c}\left(\mathbf{f}^{i}(t)\right)+\alpha \mathbf{b}\left(\mathbf{f}^{i}(t)\right) .
\end{aligned}
$$

Here prime denotes differentiation with respect to time, $g$ is the concentration of TGF $\beta, s$ is the speed of the cell and is a function of fiber density and $g, \tau$ a time lag and the superscript $i$ identifies the cell. The parameters $\rho$ and $\alpha$ are positive constants. These equations model the contact guidance of the collagen and fibrin matrices as well as the cells directional persistence due to cell polarization. When $\rho=0$ the cell are not polarized and move only in the direction determined by the extracellular matrix. When $\alpha=0$ the fibroblast direction is determined entirely by the collagen component of the extracellular matrix. The cell polarization parameter does not dramatically affect the collagen alignment and in all the simulations shown we take $\rho=0.9$, reflecting the high degree of polarity in fibroblasts on fibrous gels. The value for $\alpha$ is chosen to be 0.5 assuming that the fibrin clot and the collagen matrix would equally influence the direction of the fibroblasts.

Each fibroblast in the simulation can contribute to the reordering of the collagen. We define a vector f as

$$
\mathbf{f}(\mathbf{x}, t)=\sum_{i=0}^{M} w\left(\mathbf{f}^{i}(t)-\mathbf{x}\right) \frac{\mathbf{f}^{i}(t-\tau)^{\prime}}{\left\|\mathbf{f}^{i}(t-\tau)^{\prime}\right\|}
$$

where $\tau$ again represents a time lag. This gives the cumulative affect of all the fibroblasts on the collagen direction with the weight function, $w$, keeping the influence of the fibroblasts local. The orientation of $\mathbf{c}(\mathbf{x})$ evolves according to

$$
\frac{d \theta(t)}{d t}=\kappa\|\mathbf{f}\| \sin (\phi-\theta)
$$

where $\theta$ is the direction of the collagen matrix at a point in space, $\kappa$ is a parameter which measures the ability of the fibroblasts to reorder the matrix and $\phi$ is the direction of the vector $\mathbf{f}$. This functional 
form causes the derivative to be small when the difference between the angles is small, maximal when the difference is 90 degrees and it is periodic so when the angles are close to 180 degrees apart the derivate is again small. The orientation of $\mathbf{b}(\mathbf{x})$ is given in the initial conditions. The densities of the proteins are given by the lengths of their representative vectors which are governed by the following ordinary differential equations

$$
\begin{aligned}
\frac{d\|\mathbf{c}\|}{d t} & =\left(p_{c}-d_{c}\|\mathbf{c}\|\right) \sum_{i=1}^{M} w\left(\mathbf{f}^{i}-\mathbf{x}\right) \\
\frac{d\|\mathbf{b}\|}{d t} & =-d_{b}\|\mathbf{b}\| \sum_{i=1}^{M} w\left(\mathbf{f}^{i}-\mathbf{x}\right)
\end{aligned}
$$

where $p_{c}, d_{c}$ and $d_{b}$ are positive functions which depend on the concentration of TGF $\beta$. Thus we have assumed the degradation rate for collagen and fibrin which is proportional to the corresponding protein density. 